\title{
A VARIATIONAL WAVE ACQUISITION STEREO SYSTEM FOR THE 3-D RECONSTRUCTION OF OCEANIC SEA STATES
}

\author{
Guillermo Gallego \\ School of Electrical and Computer Engineering \\ Georgia Institute of Technology, Atlanta, USA.
}

Francesco Fedele

School of Civil and Environmental Engineering

Georgia Institute of Technology, Savannah, USA.

\author{
Anthony Yezzi \\ School of Electrical and Computer Engineering \\ Georgia Institute of Technology, Atlanta, USA.
}

\author{
Alvise Benetazzo \\ CNR-ISMAR, Venice, Italy.
}

\begin{abstract}
We propose a novel remote sensing technique that infers the three-dimensional wave form and radiance of oceanic sea states via a variational stereo imagery formulation. In this setting, the shape and radiance of the wave surface are minimizers of a composite cost functional which combines a data fidelity term and smoothness priors on the unknowns. The solution of a system of coupled partial differential equations derived from the cost functional yields the desired ocean surface shape and radiance. The proposed method is naturally extended to study the spatiotemporal dynamics of ocean waves, and applied to three sets of video data. Statistical and spectral analysis are carried out. The results shows evidence of the fact that the omni-directional wavenumber spectrum $S(k)$ of the reconstructed waves decays as $k^{-2.5}$ in agreement with Zakharov's theory (1999). Further, the three-dimensional spectrum of the reconstructed wave surface is exploited to estimate wave dispersion and currents.
\end{abstract}

\section{INTRODUCTION}

Wind-generated waves play a prominent role at the interfaces of the ocean with the atmosphere, land and solid Earth. Waves also define in many ways the appearance of the ocean seen by remote-sensing instruments. Classical observational methods rely on time series retrieved from wave gauges and ultrasonic instruments or buoys to measure the space-time dynamics of ocean waves. Global altimeters, or Synthetic Aperture Radar (SAR) instruments are exploited for observations of large oceanic ar- eas via satellites, but details on small-scales are lost. Herein, we propose to complement the abovementioned instruments with a novel video observational system which relies on variational stereo techniques to reconstruct the 3-D wave surface both in space and time. Such system uses two or more stereo camera views pointing at the ocean to provide spatio-temporal data and statistical content richer than that of previous monitoring methods. Vision systems are non-intrusive and have economical advantages over their predecessors, but they require more processing power to extract information from the ocean.

Since this work covers both the topics of shape reconstruction and oceanic sea states, it relates to a vast body of literature. The three-dimensional reconstruction of an object's surface from stereo pairs of images is a classical problem in computer vision (see, for example [1-4]), and it is still an extremely active research area. There are many 3-D reconstruction algorithms available in the literature and the reconstruction problem is far from being solved. The different algorithms are designed under different assumptions and provide a variety of trade-offs between speed, accuracy and viability. Traditional image-based stereo methods typically consist of two steps: first image points or regions are detected and matched across images by optimizing a photometric score to establish local correspondences; then depth is inferred by combining these correspondences using triangulation of 3-D points (back-projection of image points). The first step, also known as the stereo matching problem, is significantly more difficult than the second one. However, epipolar geometry between image pairs can be exploited to reduce stereo 
matching to a 1-D search along epipolar lines. This is the strategy used in recent systems $[5,6]$. This approach has the advantages of being simple and fast. However, it also has some major disadvantages that motivated the research on improved stereo reconstruction methods [7-9]. These disadvantages are: (i) Correspondences rely on strong textures (high contrast between intensities of neighboring points) and image matching gives poor correspondences if the objects in the scene have a smooth radiance. Correspondences also suffer from the presence of noise and local minima. (ii) Each space point is reconstructed independently and therefore the recovered surface of an object is obtained as a collection of scattered 3-D points. Thus, the hypothesis of the continuity of the surface is not exploited in the reconstruction process. The breakdown of traditional stereo methods in these situations is evidenced by "holes" in the reconstructed surface, which correspond to unmatched image regions $[1,5]$. This phenomenon may be dominant in the case of the ocean surface, which, by nature, is generally continuous and contains little texture.

Modern object-based image processing and computer vision methods that rely on Calculus of Variations and Partial Differential Equations (PDEs), such as Stereoscopic Segmentation [8] and other variational stereo methods $[7,9,10]$, are able to overcome the disadvantages of traditional stereo. For instance, unmatched regions are avoided by building an explicit model of the smooth surface to be estimated rather than representing it as a collection of scattered 3-D points. Thus, variational methods provide dense and coherent surface reconstructions. Surface points are reconstructed by exploiting the continuity (coherence) hypothesis in the full two-dimensional domain of the surface. Variational stereo methods combine correspondence establishment and shape reconstruction into one single step and they are less sensitive to matching problems of local correspondences. The reconstructed surface is obtained by minimization of an energy functional designed for the stereo problem. The solution is obtained in the context of active surfaces by deforming an initial surface via a gradient descent PDE derived from the necessary optimality conditions of the energy functional, the so-called Euler-Lagrange (EL) equations.

In the context of oceanography, the first experiments with stereo cameras mounted on a ship were by Schumacher [11] in 1939. Later, Coté et al. [12] in 1960 demonstrated the use of stereo-photography to measure the sea topography for long ocean waves. The study of long waves using stereophotography was also discussed by Sugimori [13], based on an optical method by Barber [14], and by Holthuijsen [15]. Stereography gained popularity in studying the dynamics of oceanographic phenomena during the 1980s due to advances in hardware. Shemdin et al. $[16,17]$ applied stereography for the directional measurement of short ocean waves. In 1997, Holland et al. [18] demonstrated the practical use of video systems to measure nearshore physical processes. A more recent integration of stereographic techniques into the field of oceanography has been the WAVESCAN project

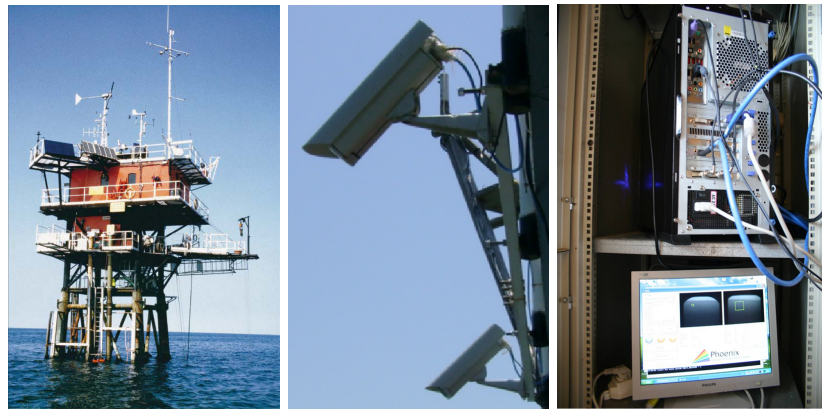

FIGURE 1. Left: off-shore platform "Acqua Alta" in the Northern Adriatic Sea, near Venice. Center: pair of synchronized cameras for monitoring the ocean climate from the platform. Right: WASS hardware installed at the platform for recording stereo videos of ocean waves.

of Santel et al. [19].

Recently, Benetazzo [5] successfully incorporated epipolar techniques in the Wave Acquisition Stereo System (WASS). This was tested in experiments off the shore of the California Coast and the Venice coast in Italy. Benetazzo was able to estimate wave spectra from the extracted time series of the surface fluctuations at one fixed point given the data images. The accuracy of such spectral estimates is comparable to the accuracy obtained from ultrasonic transducer measurements. An example of a WASS system currently installed at the Acqua Alta platform is shown in Fig. 1. An alternative trinocular imaging system (ATSIS) for measuring the temporal evolution of 3-D surface waves was proposed in [6]. More recently, in [20] it is shown how a modern variational stereo reconstruction technique pioneered by [7] can be applied to the estimation of oceanic sea states. Additional references demonstrate that this is an active research topic [21-24].

Encouraged by the results in $[5,20,25]$, in this paper we propose a novel variational framework for the recovery of the shape of ocean waves given multi-view stereo imagery. In particular, motivated by the characteristics of the target object in the scene, i.e., the ocean surface, we first introduce the graph surface representation in the formulation of the reconstruction problem. Then, we present the new variational stereo method in the context of active surfaces. The performance of the algorithm is validated on experimental data collected off shore, and the statistics of the reconstructed surface are also analyzed. Concluding remarks and future research directions are finally presented.

\section{THE VARIATIONAL GEOMETRIC METHOD}

This paper is inspired by the works of $[5,20]$ and [8]. In particular, the variational approach of Stereoscopic Segmentation [8] is used to tackle the vision problem: the reconstructed surface of the ocean is obtained as the minimizer of an energy functional designed to fit the measurements of ocean waves. In 
every 3-D reconstruction method, the quality and accuracy of the results depend on the calibration of the cameras. There are standard camera calibration procedures in the literature to characterize accurately the intrinsic and extrinsic parameters of the cameras [1]. We assume cameras are calibrated and synchronized, and we focus on the reconstruction of the water surface for a fixed time.

\section{Multi-image setup. Graph surface representation}

Let $S$ be a smooth surface in $\mathbb{R}^{3}$ with generic local coordinates $(u, v) \in \mathbb{R}^{2}$. Let $\left\{I_{i}\right\}_{i=1}^{N_{c}}$ be a set of images of a static (water) scene acquired by cameras whose calibration parameters are $\left\{\mathrm{P}^{i}\right\}_{i=1}^{N_{c}}$. Space points are mapped into image points according to the pinhole camera model [2]. The equations of such a perspective projection mapping are linear if expressed in homogeneous coordinates of Projective geometry. A surface point (or, in general a 3D point) $\mathbf{X}=(X, Y, Z)^{\top}$ with homogeneous coordinates $\overline{\mathbf{X}}=(X, Y, Z, 1)^{\top}$ is mapped to point $\mathbf{x}_{i}=\left(x_{i}, y_{i}\right)^{\top}$ in the $i$-th image with homogeneous coordinates $\overline{\mathbf{x}}_{i}=\left(x_{i}, y_{i}, 1\right)^{\top} \sim \mathrm{P}^{i} \overline{\mathbf{X}}$, where the symbol $\sim$ means equality up to a nonzero scale factor and $\mathrm{P}^{i}=\mathrm{K}^{i}\left[\mathrm{R}^{i} \mid \mathbf{t}^{i}\right]$ is the $3 \times 4$ projection matrix with the intrinsic $\left(\mathrm{K}^{i}\right)$ and extrinsic $\left(\mathrm{R}^{i}, \mathbf{t}^{i}\right)$ calibration parameters of the $i$-th camera. These parameters are known under the hypothesis of calibrated cameras. The optical center of the camera is the point $\mathbf{C}_{i}=\left(C_{i}^{1}, C_{i}^{2}, C_{i}^{3}\right)^{\top}$ that satisfies $\mathrm{P}^{i} \overline{\mathbf{C}}_{i}=\mathbf{0}$. Let $\pi_{i}: \mathbb{R}^{3} \rightarrow \mathbb{R}^{2}$ note the projection maps: $\mathbf{x}_{i}=\pi_{i}(\mathbf{X})$. Finally, $I_{i}\left(\mathbf{x}_{i}\right) \equiv I_{i}\left(\pi_{i}(\mathbf{X})\right)$ is the intensity at $\mathbf{x}_{i}$.

We present a different approach to the reconstruction problem discussed in $[7,8]$ by exploiting the hypothesis that the surface of the water can be represented in the form of a graph or elevation map:

$$
Z=Z(X, Y)
$$

where $Z$ is the height of the surface with respect to a domain plane that is parameterized by coordinates $X$ and $Y$. Indeed, slow varying, non-breaking waves admit this simple representation with respect to a plane orthogonal to gravity direction. As a natural extension of existing variational stereo methods, energy functionals can be tailored to exploit the benefits of this valuable representation. The surface can still be obtained as the minimizer of a suitable energy functional but now with a different geometrical representation of the solution.

The graph representation of the water surface presents some clear advantages over the more general level set representation of $[7-9,20]$. Surface evolution is simpler to implement since the surface is not represented in terms of an auxiliary higher dimensional function (the level set function). The surface is evolved directly via the height function (1) discretized over a fixed 2-D grid defined on the $X-Y$ plane. The latter also implies that for the same amount of physical memory, higher spatial resolution (finer details) can be achieved in the graph representation than with the level set. The $X-Y$ plane becomes the natural common domain to parameterize the geometrical and photometric properties of surfaces. This simple identification does not exist in the level set approach [8]. Finally, the graph representation allows for fast numerical solvers besides gradient descent, like Fast Poisson Solvers, Cyclic Reduction, Multigrid Methods, Finite-Element Methods (FEM), etc. In the level set framework, the range of solvers is not as diverse.

However, there are also some minor disadvantages. A world frame properly oriented with the gravity direction must be defined in advance to represent the surface as a graph with respect to this plane. This is not trivial a priori and might pose a problem if only the information from the stereo images is used [5]. This condition may not be so if external gravity sensors provide this information. Surface evolution is constrained to be in the form of a graph and this may not be the same as the evolution described for an unconstrained surface. As a result, more iterations may be required to reach convergence.

The reconstruction problem is mathematically stated in the following section. The desired surface is given by the solution of a variational optimization problem.

\section{Proposed energy functional}

Consider the 3-D reconstruction problem from a collection of $N_{c} \geq 2$ input images (we will exemplify with $N_{c}=2$ ). We investigate a generative model of the images that allows for the joint estimation of the shape of the surface $S$ and the radiance function on the surface $f$ as minimizers of an energy functional. Let the energy functional be the weighted sum of a data fidelity term $E_{\text {data }}$ and two regularizing terms: a geometry smoothing term $E_{\text {geom }}$ and a radiance smoothing term $E_{\text {rad }}$,

$$
E(S, f)=E_{\mathrm{data}}(S, f)+\alpha E_{\mathrm{geom}}(S)+\beta E_{\mathrm{rad}}(f),
$$

where $\alpha, \beta \in \mathbb{R}^{+}$. The data fidelity term measures the photoconsistency of the model: the discrepancy in the $L^{2}$ sense between the observed images $I_{i}$ and the radiance model $f$,

$$
E_{\mathrm{data}}=\sum_{i=1}^{N_{c}} E_{i}, \quad E_{i}=\int_{\Omega_{i}} \phi_{i} \mathrm{~d} \mathbf{x}_{i},
$$

where the photometric matching criterion is

$$
\phi_{i}=\frac{1}{2}\left(I_{i}\left(\mathbf{x}_{i}\right)-f\left(\mathbf{x}_{i}\right)\right)^{2} .
$$

The region of the image domain where the scene is projected is denoted by $\Omega_{i}$. The meaning of $f\left(\mathbf{x}_{i}\right)$ will be clear shortly. 
Assuming that the surface of the scene is represented as a graph $Z=Z(u, v)$, a point on the surface has coordinates

$$
\mathbf{X}(u, v)=(u, v, Z(u, v))^{\top}
$$

The chain of operations to obtain the intensity $I_{i}\left(\mathbf{x}_{i}\right)$ given a surface point with world coordinates $\mathbf{X}(\mathbf{u}) \equiv S(\mathbf{u}), \mathbf{u}=(u, v)^{\top}$, is

$$
\mathbf{X}(\mathbf{u}) \mapsto \tilde{\mathbf{X}}_{i}=\mathrm{M}^{i} \mathbf{X}+\mathbf{p}_{4}^{i} \mapsto \mathbf{x}_{i} \mapsto I_{i}\left(\mathbf{x}_{i}\right),
$$

where $\tilde{\mathbf{X}}^{i}=\left(\tilde{X}_{i}, \tilde{Y}_{i}, \tilde{Z}_{i}\right)^{\top}$ are related to the coordinates of $\mathbf{X}$ in the $i$-th camera frame, $\mathbf{x}_{i}=\left(x_{i}, y_{i}\right)^{\top}=\left(\tilde{X}_{i} / \tilde{Z}_{i}, \tilde{Y}_{i} / \tilde{Z}_{i}\right)^{\top}$ are the coordinates of the projection of $\mathbf{X}$ in the $i$-th image plane and $\mathrm{P}^{i}=\left[\mathrm{M}^{i} \mid \mathbf{p}_{4}^{i}\right]$ is the projection matrix of the camera corresponding to the $i$-th image, in world coordinates, i.e., $\mathrm{M}^{i}=\mathrm{K}^{i} \mathrm{R}^{i} \equiv$ $\left(\mathbf{n}_{1}^{i}, \mathbf{n}_{2}^{i}, \mathbf{n}_{3}^{i}\right)^{\top}$ and $\mathbf{p}_{4}^{i}=K^{i} \mathbf{t}^{i}$. Also, $\left|\mathrm{M}^{i}\right|=\operatorname{det}\left(\mathrm{M}^{i}\right)$.

The radiance model $f$ is specified by a function $\hat{f}$ defined on the surface $S$. Then, $f$ in (4) is naturally defined by $f\left(\mathbf{x}_{i}\right)=$ $\hat{f}\left(\pi_{i}^{-1}(\mathbf{X})\right)$, where $\pi_{i}^{-1}$ denotes the back-projection operation from a point in the $i$-th image to the closest surface point with respect to the camera. With a slight abuse of notation, let us use $f$ to denote the parameterized radiance $f(\mathbf{u})$, understanding that $f\left(\mathbf{x}_{i}\right)$ in (4) reads the back-projected value in $\hat{f}(\mathbf{X}(\mathbf{u}))=f(\mathbf{u})$.

Motivated by the common parameterizing domain of the shape and radiance of the surface and to obtain the simplest diffusive terms in the PDEs derived from the necessary optimality conditions of the energy (2), let the regularizers be

$$
\begin{aligned}
E_{\text {geom }} & =\int_{U} \frac{1}{2}\|\nabla Z(\mathbf{u})\|^{2} \mathrm{~d} \mathbf{u}, \\
E_{\mathrm{rad}} & =\int_{U} \frac{1}{2}\|\nabla f(\mathbf{u})\|^{2} \mathrm{~d} \mathbf{u},
\end{aligned}
$$

where $\nabla Z=\left(Z_{u}, Z_{v}\right)^{\top}, \nabla f=\left(f_{u}, f_{v}\right)^{\top}$ and subscripts indicate the derivative with respect to that variable. Once all terms in (2) have been specified, some transformations are carried out to express the data fidelity integrals over a more suitable domain: the parameter space. The Jacobian of the change of variables between integration domains is, by applying the chain rule to (6),

$$
\mathrm{J}_{i}=\left|\frac{\mathrm{d} \mathbf{x}_{i}}{\mathrm{~d} \mathbf{u}}\right|=-\left|\mathrm{M}^{i}\right| \tilde{Z}_{i}^{-3}\left(\mathbf{X}-\mathbf{C}_{i}\right) \cdot\left(\mathbf{X}_{u} \times \mathbf{X}_{v}\right),
$$

where $\mathbf{X}_{u} \times \mathbf{X}_{v}$ is proportional to the outward unit normal $\mathbf{N}$ to the surface at $\mathbf{X}(u, v)$, and $\tilde{Z}_{i}=\mathbf{n}_{3}^{i} \cdot\left(\mathbf{X}-\mathbf{C}_{i}\right)>0$ is the depth of the point $\mathbf{X}$ with respect to the $i$-th camera (located at $\mathbf{C}_{i}$ ). With this change, energy (3) becomes

$$
E_{i}=\int_{\Omega_{i}} \phi_{i} \mathrm{~d} \mathbf{x}_{i}=\int_{U} \phi_{i} \mathrm{~J}_{i} \mathrm{~d} \mathbf{u},
$$

where the last integral is over $U$ : the part of the parameter space whose surface projects on $\Omega_{i}$ in the $i$-th image. Observe that the Jacobian weights the photometric error $\phi_{i}$ proportionally to the cosine of the angle between the unit normal to the surface at $\mathbf{X}$ and the projection ray (the ray joining the optical center of the camera and $\mathbf{X})$ : $\left(\mathbf{X}-\mathbf{C}_{i}\right) \cdot\left(\mathbf{X}_{u} \times \mathbf{X}_{v}\right)$. After collecting terms (7), (8), and (10), and noting that the shape $\mathbf{X}$ of the surface solely depends on the height (Eqn. (5)), energy (2) becomes the integral of the so-called Lagrangian L:

$$
E(Z, f)=\int_{U} L\left(Z, Z_{u}, Z_{v}, f, f_{u}, f_{v}, u, v\right) \mathrm{d} \mathbf{u} .
$$

\section{Energy minimization. Optimality condition}

The energy (11) depends on two functions: the shape $Z$ and the radiance $f$ of the surface. To find a minimizer of such a functional, we derive the necessary optimality condition by setting to zero the first variation of the functional. Using standard techniques from Calculus of Variations, the first variation (Gâteaux derivative) of (11) has two terms: one in the interior of the integration region $U$ in the parameter space and one boundary term (on $\partial U$ ). Setting the first variation to zero for all possible smooth perturbations yields a coupled system of PDEs (EL equations) along with natural boundary conditions:

$$
\begin{array}{rlrl}
g(Z, f)-\alpha \Delta Z & =0 & & \text { in } U, \\
b(Z, f)+\alpha \frac{\partial Z}{\partial v}=0 & & \text { on } \partial U, \\
-\sum_{i=1}^{N_{c}}\left(I_{i}-f\right) \mathrm{J}_{i}(Z)-\beta \Delta f=0 & & \text { in } U, \\
\beta \frac{\partial f}{\partial v}=0 & & \text { on } \partial U,
\end{array}
$$

where the non-linear terms due to the data fidelity energy are

$$
\begin{aligned}
& g(Z, f)=\nabla f \cdot \sum_{i=1}^{N_{c}}\left|\mathbb{M}^{i}\right| \tilde{Z}_{i}^{-3}\left(I_{i}-f\right)\left(u-C_{i}^{1}, v-C_{i}^{2}\right), \\
& b(Z, f)=\sum_{i=1}^{N_{c}} \phi_{i}\left|\mathbb{M}^{i}\right| \tilde{Z}_{i}^{-3}\left(\left(u-C_{i}^{1}\right) v^{u}+\left(v-C_{i}^{2}\right) v^{v}\right) .
\end{aligned}
$$

The Laplacians $\Delta Z$ and $\Delta f$ arise from the regularizing terms (7) and (8), respectively, and $\partial * / \partial v$ is the usual notation for the directional derivative along $v=\left(v^{u}, v^{v}\right)^{\top}$, the normal to the integration domain $U$ in the parameter space.

A simple classification of the PDEs can be done as follows. For a fixed surface, (14) and (15) form a linear elliptic PDE (of the inhomogeneous Helmholtz type) with Neumann boundary conditions. On the other hand, for a fixed radiance, (12) and (13) lead to a nonlinear elliptic equation in the height $Z$ with nonstandard boundary conditions.

A common approach to solve difficult EL equations, such as the EL equation presented in (12)-(15), is to add an artificial 
time marching variable $t$ dependency in the unknown functions (height, radiance) and set up a gradient descent flow that will drive their evolution such that the energy (11) will decrease in time. Thus the solution of the EL equations is obtained as the steady-state of the gradient descent equations. This is the context of the so-called active surfaces. The gradient descent PDEs are:

$$
\begin{aligned}
Z_{t} & =\alpha \Delta Z-g(Z, f), \\
f_{t} & =\beta \Delta f-\sum_{i=1}^{N_{c}} \mathrm{~J}_{i}(Z) f+\sum_{i=1}^{N_{c}} I_{i} \mathrm{~J}_{i}(Z) .
\end{aligned}
$$

To simplify the equations, we approximate the boundary condition (13) by a simpler, homogeneous Neumann boundary condition. This can be interpreted as if the data fidelity term vanished close to the boundary and it is a reasonable assumption since the major contribution to the energy is given by the terms in $U$, not at the boundary.

\section{Numerical solution}

An iterative, alternating approach is used to find the minimum of energy (2) via the evolution of the coupled gradient descent PDEs (17)-(18). During each iteration there are two phases: (i) evolve the shape, leaving the radiance fixed, and (ii) evolve the radiance, leaving the shape unchanged. The PDEs are discretized on a rectangular 2-D grid in the parameter space and then solved numerically using finite-difference methods (FDM). Forward differences in time and central differences in space approximate the derivatives, yielding an explicit updating scheme. The time step $\Delta t$ in the scheme is determined by the stability condition of the resulting PDE. For the linear PDE (18), the time step for $\ell^{2}$ stability satisfies $\Delta t \leq 1 /\left(\frac{4 \beta}{h^{2}}+\frac{1}{2} \max \sum_{k=1}^{N_{c}} \mathrm{~J}_{k}\right)$, where $h$ is the spatial step size of the grid, $\mathrm{J}_{k}(Z) \geq 0$ and the maximum is taken over the 2-D discretized Jacobians for the current height function. The time step may change at every iteration, depending on the value of the evolving height. For the nonlinear PDE (12), the von Neumann stability analysis of the linearized PDE yields a time step $\Delta t \leq 1 /\left(\frac{4 \alpha}{h^{2}}+\frac{1}{2} \max |\dot{g}(Z)|\right)$, where $\dot{g}(Z)$ is the derivative of (16) and the maximum is taken over the 2-D discretized grid at the current time.

The previous time-stepping methods are used as relaxation procedures inside a multigrid method [26] that approximately solves the EL equations. Multigrid methods are the most efficient numerical tools for solving elliptic boundary value problems.

\section{EXPERIMENTS}

Experiment 1. Images of "Canale della Giudecca" in Venice (Italy). After validating the numerical implementation of the proposed variational stereo method with synthetic data, some experiments with real data are carried out. Figs. 2, 3 and 4 show an example of a reconstructed water surface from images of the

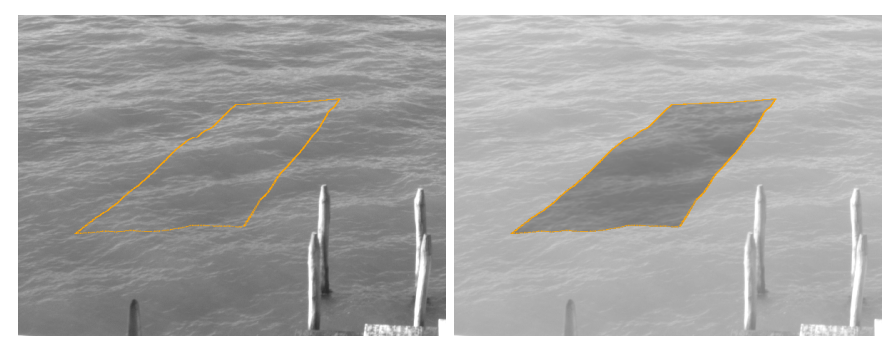

FIGURE 2. Experiment I (Venice). Left: projection of the boundary of the estimated graph, which has been discretized on a grid of $129 \times$ 513 points. Right: modeled image (computed from surface height and radiance) superimposed on original image.
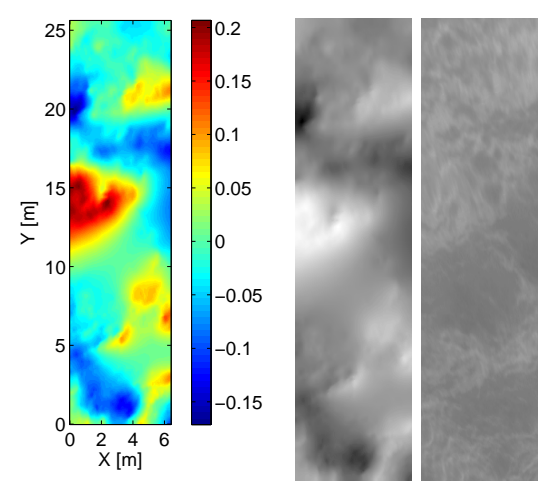

FIGURE 3. Experiment I (Venice). Left: estimated height function $Z(u, v)$ (shape of the water surface) in pseudo-color. Center: height function represented by grayscale intensities, from dark (low) to bright (high). Right: estimated radiance function $f(u, v)$, i.e., texture on the surface.

Venice Canal. Cropped images in Fig. 2 are of size $600 \times 450$ pixels and show the region of interest to be reconstructed. Fig. 2 also displays one of the modeled images created by the generative model within our variational method. The data fidelity term compares the intensities of the original and modeled images in the highlighted region, in all images. As observed, the modeled image is a good match of the original image. Fig. 3 shows the converged values of the unknowns of the problem (the height and the radiance of the surface), while Fig. 4 shows the 3-D representation of the reconstructed surface obtained by combining both 2-D functions from Fig. 3. In this experiment, the values of the weights of the regularizers were empirically determined: $\alpha=0.035$ and $\beta=0.01$. At the finest of the 5-level multigrid [26] algorithm, the gradient descent PDEs are discretized on a 2 -D grid with $129 \times 513$ points. The distance between adjacent grid points is $h=5 \mathrm{~cm}$. Therefore, the grid covers an area of $6.45 \times 25.65 \mathrm{~m}^{2}$. An example of a surface discretized at the finest grid level is shown in Fig. 4. Observe the high density of the sur- 

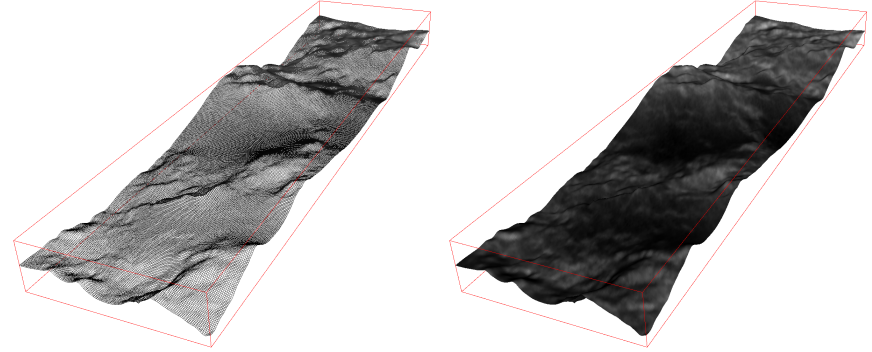

FIGURE 4. Experiment I (Venice). Left: perspective, threedimensional wire-frame representation of the estimated surface shape (height) according to grid points. Right: texture-mapped surface obtained by incorporating the radiance function in the wire-frame model. The vertical axis has been magnified by a factor of 5 with respect to the horizontal axes for visualization purpose.

face representation, typical of variational methods. The step size $h$ must be chosen so that it approximately matches the resolution in the images: a displacement of 1 pixel is observable at the finest grid level in the multigrid framework and it corresponds to a physical displacement of at least $h$. Due to perspective projection, the maximum value of $h$ is determined by the grid points closest to the cameras.

Experiment 2. Image sequence I. The method proposed in this paper is naturally extended to process stereo video on a snapshotby-snapshot basis by estimating the new surface shape and radiance based on the previously reconstructed surface. This sequential processing is the simplest way in which the method can be applied to stereo video imagery. We test the method on a different video data consisting of 10 consecutive snapshots (frames) with images of size $1000 \times 1000$ pixels. A grid of size $513 \times 513$ points and with a step size $h=1.5 \mathrm{~cm}$ is selected. Thus, the grid covers an area of $7.7 \times 7.7 \mathrm{~m}^{2}$. The deforming surface is initialized by the plane $Z=0$. A multigrid method with 6 levels and $200 \mathrm{~V}$-cycles (with 1 pre- and post-relaxation sweeps per level) is used to solve the problem at each snapshot. For the first frame, a full multigrid method (FMG) with $200 \mathrm{~V}$-cycles per level is performed prior to entering the above processing schedule. In this experiment, the weights of the regularizers are $\alpha=4 \cdot 10^{-2}$ and $\beta=4 \cdot 10^{-3}$. A reconstruction of the wave surface from video data collected by Benetazzo [5] is shown in Fig. 5. In the same figure we also report the the omni-directional spectrum $S(k)$ (averaged over the frames), computed by numerically integrating the 2-D spectrum $S\left(k_{1}, k_{2}\right)$ of the elevation map over all directions. In agreement with Zakharov's theory [27], the spectrum tail decays close to $k^{-2.5}$, where $k$ is the wave number.

Experiment 3. Image sequence II. We apply our variational method to a sequence of 2000 snapshots acquired at $10 \mathrm{~Hz}$ and at an off-shore platform near the southern seashore of the Crimean
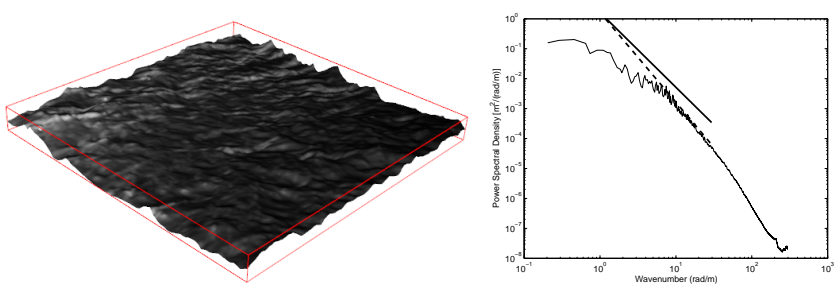

FIGURE 5. Experiment II (San Diego). Left: Surface reconstruction from a snapshot of the data in [5]. Right: Average omni-directional wave number spectrum. Straight lines: $k^{-2.5}$ (solid), $k^{-3}$ (dashed).
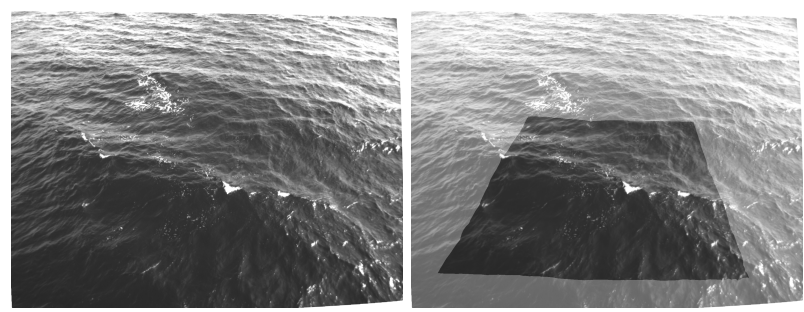

FIGURE 6. Experiment III (Crimea): Original image (left) and modeled image superimposed on original image (right).

peninsula, in the Black Sea. Two cameras mounted $12 \mathrm{~m}$ above the mean sea level and with a baseline of $2.5 \mathrm{~m}$ acquire images of size $1624 \times 1236$ pixels. Fig. 6 (left) shows a sample image from one of the cameras. A grid with $513 \times 513$ points and resolution $h=2.5 \mathrm{~cm}$, covering an area of $13 \times 13 \mathrm{~m}^{2}$, is used to discretize the graph of the surface. Fig. 6 (right) shows the approximate region of interest occupied by the projection of the reconstructed surface on one of the images. Roughly, 1 image pixel corresponds to a physical displacement of $1.06 \mathrm{~cm}(1.88 \mathrm{~cm})$ for grid points near (resp. far from) the cameras. Both displacements are of the same order as $h$. The same multigrid processing scheme as in experiment 2 is used, but with $1000 \mathrm{~V}$-cycles per level. The weights of the regularizers are $\alpha=0.1$ and $\beta=0.025$.

The four-dimensional reconstructed wave surface can be represented in the form of a space-time volume of wave heights, $V=Z(x, y, t)$, as visualized in Figure 7, where the oscillating pattern of the waves is evident by the oscillating color patterns. The spectra and statistics of the waves can be computed from the reconstructed surface.

The mean omni-directional spectrum $S(k)$, averaged over all 2000 snapshots, is shown in Fig. 8. According to the wave turbulence theory of Zakharov [27], the spectrum tail initially decays as $k^{-2.5}$ as a result of an energy cascade from large to small scales up to $\sim 10 \mathrm{rad} / \mathrm{m}$ and then switch to a $k^{-3}$ equilibrium range [28]. Fig. 8 clearly shows such behavior of the tail of the spectrum. More generally, Fourier analysis can be applied directly to the wave space-time volume $Z(x, y, t)$, resulting in a 3 -D spectrum $\mathscr{Z}\left(k_{x}, k_{y}, \omega\right)$, where frequencies $k_{x}, k_{y}$ 

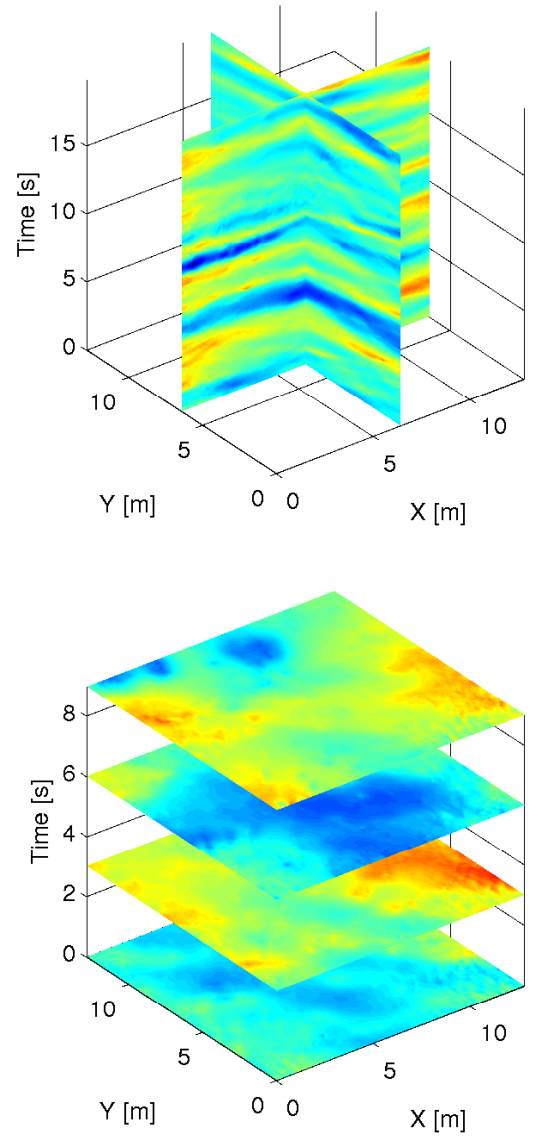

FIGURE 7. Experiment III (Crimea). Vertical (Top) and Horizontal (Bottom) slicing of the wave space-time volume $Z(x, y, t)$ : reconstructions for fixed $x$ - $t$ or $y$ - $t$ planes. The wave heights $Z$ have been pseudocolored from blue (low) to red (high).

and $\omega$ are the Fourier transformed variables of $x, y$ and $t$, respectively. Recall that $\omega=2 \pi f$ is the angular frequency and $f \equiv f_{t}$ is the linear frequency, measured in Hertzs. Similarly, let us use $f_{x}=k_{x} /(2 \pi)$ and $f_{y}=k_{y} /(2 \pi)$ for the wave numbers in units of cycles $/ \mathrm{m}$. The 3-D spectrum contains information of the propagation characteristics of the waves, such as their wavelengths, frequencies, and their directions and speeds of propagation. From a practical point of view, the 3-D spectrum of the reconstructed $513 \times 513 \times 2000$ wave height grid is computed by averaging the 3-D spectra of non-overlapping pieces of the grid. We split the wave space-time volume along the temporal dimension to compute the 3-D spectrum on a Fourier grid with $512 \times 512 \times 512$ points; thus, each piece consists of $N_{t}=$ 512 snapshots. The Nyquist wave numbers are $\left[f_{x}, f_{y}, f\right]_{\max }=$ $\left[h^{-1}, h^{-1}, \Delta t^{-1}\right]=[20$ cycles $/ m, 20$ cycles $/ m, 5 \mathrm{~Hz}]$. The spectral resolutions are given by $\Delta f_{x}=\Delta f_{y}=1 /(N h) \approx 0.078$ cycles $/ m$
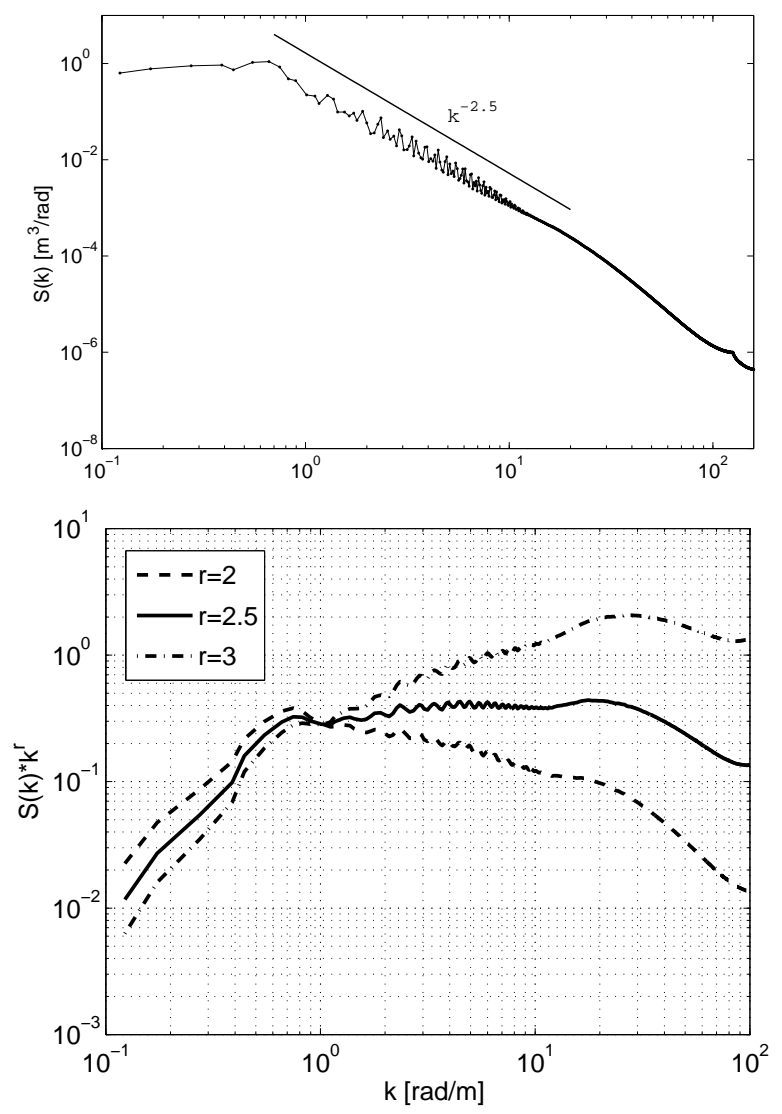

FIGURE 8. Experiment III (Crimea). Top: Mean omni-directional spectrum $S(k)$ averaged over 2000 snapshots. Bottom: mean saturation spectrum $S(k) k^{r}$ for $r=\{2,2.5,3\}$.

and $\Delta f=1 /(N \Delta t) \approx 0.02 \mathrm{~Hz}$ for the 3-D FFT with $N=512$ points in each dimension. Fig. 9 shows the 3-D wave spectrum, and Fig. 10 shows two of its slices through the frequency axes: the frequency-wave number spectra $\omega-k_{x}$ and $\omega-k_{y}$, respectively.

The white curve in the vertical slices of $\mathscr{Z}\left(k_{x}, k_{y}, \omega\right)$ corresponds to planar projections of the linear dispersion manifold in deep water, namely $\sqrt{k_{x}^{2}+k_{y}^{2}}=\omega^{2} / g$, where $g$ is gravity acceleration. Other researchers [29] have measured the $\omega-k$ spectrum for long wave ranges at nearshore events to estimate surface currents and the water depth below the waves. Their measurements are also shown in comparison to the linear dispersion relation. At the Crimean platform, the water depth is approximately 30 meters. Therefore, for all practical purposes with respect to our wave number resolution, the depth can be regarded as being infinite. The components of the current $\mathbf{v}$ can be estimated from the observed deviations from the theoretical dispersion curve, as shown in Fig. 10, by a best fit of the wave-current dispersion relation $k=(\omega-\mathbf{k} \cdot \mathbf{v})^{2} / g$, where $k=|\mathbf{k}|$ (see [30]). This yields $\mathbf{v} \approx(-0.17,-0.45) \mathrm{m} / \mathrm{s}$, with the dominant component in the 


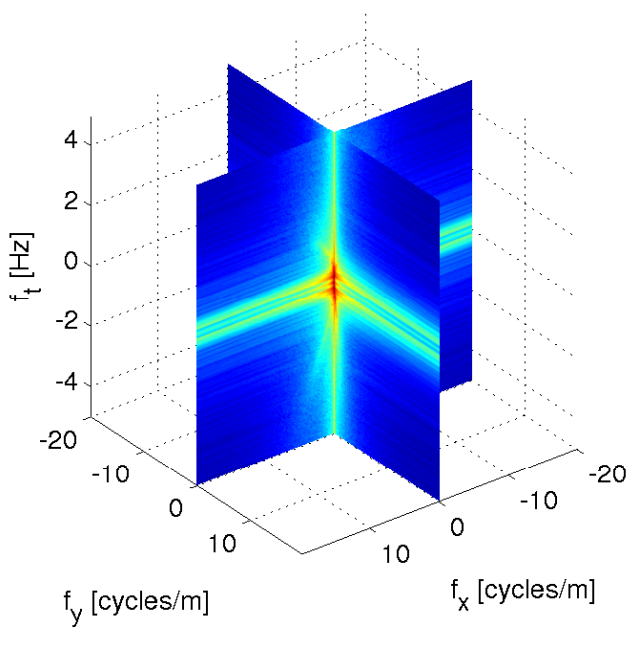

FIGURE 9. Experiment III (Crimea). Two orthogonal slices of the wave 3 -D spectrum $\mathscr{Z}\left(k_{x}, k_{y}, \omega\right)$ through the frequency origin. Slices correspond to the values of $\mathscr{Z}\left(k_{x}, k_{y}, \omega\right)$ at planes $k_{x}=0$ and $k_{y}=0$. Axes $f_{x}, f_{y}$ and $f_{t}$ stand for $k_{x} /(2 \pi), k_{y} /(2 \pi)$ and $\omega /(2 \pi)$, respectively.

$y$ direction. This propagation direction agrees with the one observed by visual inspection of the stereo video data. Figure 10 shows strong physical evidence to support the hypothesis that the variational graph method presented in this work is capturing real waves propagating in the observed direction.

Time series at virtual probes. Statistical analysis. The rich content of the space-time reconstruction of the surface wave allows for the extraction of time series of wave displacements $Z_{i}(t)=Z\left(x_{i}, y_{i}, t\right)$ from the space-time volume $V$ at virtual probes $\left(x_{i}, y_{i}\right)$ in space, as illustrated in Figure 11. Several statistical and spectral parameters that characterize the sea states can be computed from such time series. The significant wave height and mean wave period are $H_{s}=0.3 \mathrm{~m}$ and $T_{m}=2.77 \mathrm{~s}$, respectively. Fig. 12 shows the observed Power Spectral Density estimated from time series extracted from the wave space-time volume. An FFT with 2048 points was used, i.e., the spectral resolution is $\Delta f=5 \cdot 10^{-3} \mathrm{~Hz}$. If the tail of the wave number spectrum decays as $\tilde{F}(k) \propto k^{-2.5}$, the tail of the frequency spectrum decays as $F(f) \propto f^{-4}$. This behavior is observed in Figure 12, which also shows a verification of our variational method against an earlier WASS measurement technique based on epipolar geometry [5]. The peak at $2 \mathrm{~Hz}$ observed in the black curve is due to vibrations induced by fishermen walking on the Crimea platform while WASS was recording. The epipolar reconstruction [5] is purely based on the imaged data with no regularizing term as in the variational approach. The variational method unveiled the small-scale range of the spectrum improving the estimate at large wave numbers and frequencies.
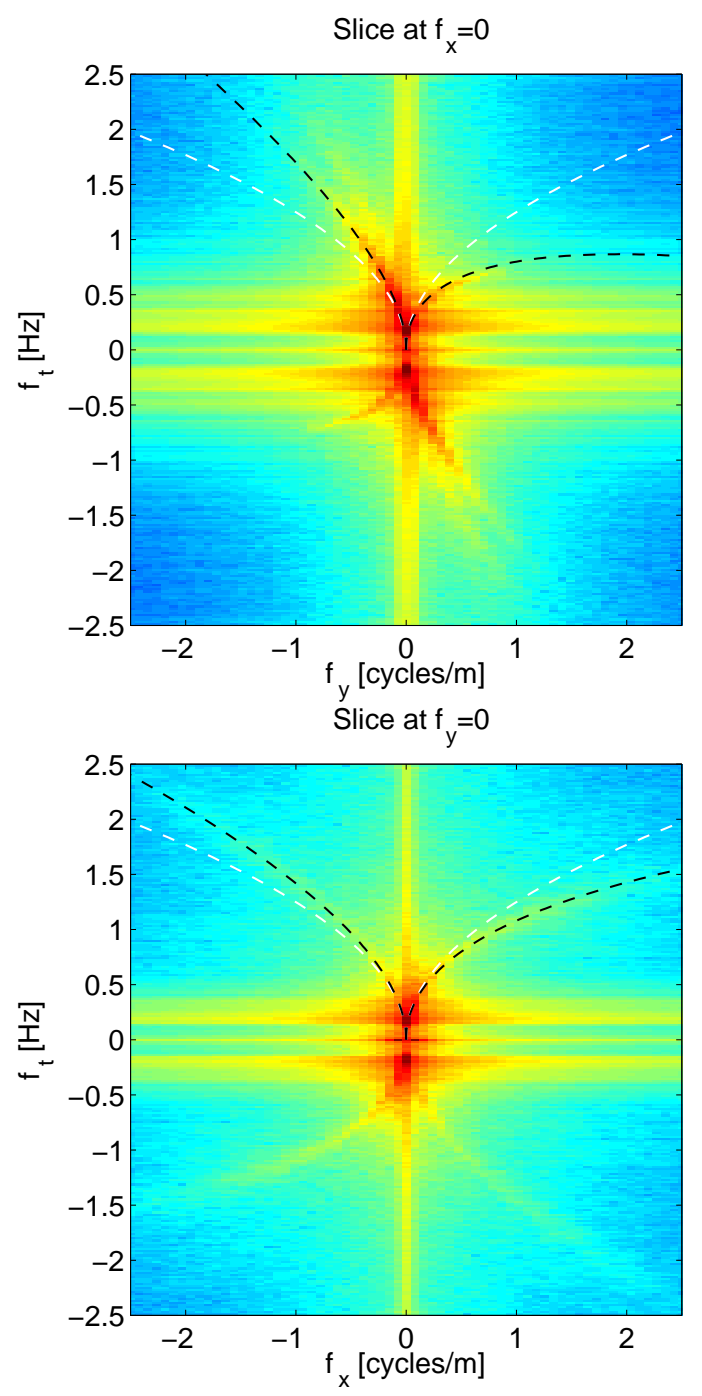

FIGURE 10. Experiment III (Crimea). Vertical slices of the 3-D wave spectrum at frequencies $k_{x}=0$ (top) and $k_{y}=0$ (bottom). Superimposed on top half of both plots: (white curve) vertical slice of the linear wave dispersion manifold $|\mathbf{k}|=\omega^{2} / g$, with $\omega=2 \pi f_{t}$, and (black curve) vertical slice of the wave-current dispersion manifold $|\mathbf{k}|=(\omega-\mathbf{k} \cdot \mathbf{v})^{2} / g$, with $\mathbf{v} \approx(-0.17,-0.45) \mathrm{m} / \mathrm{s}$. Axes $f_{x}, f_{y}$ and $f_{t}$ stand for $k_{x} /(2 \pi)$, $k_{y} /(2 \pi)$ and $\omega /(2 \pi)$, respectively.

By collecting the time waves observed at all the virtual probes indicated in Figure 11, one can estimate the wave height distribution, which is shown in Figure 13. A fair agreement with the Boccotti asymptotic form given by [31,32]

$$
P(\text { wave height }>H) \approx c \exp \left(-\frac{H^{2}}{4 \sigma^{2}\left(1+\psi^{*}\right)}\right)
$$

is observed. Here, the parameters $c$ and $\psi^{*} \equiv\left|\psi\left(T^{*}\right)\right|$ both de- 

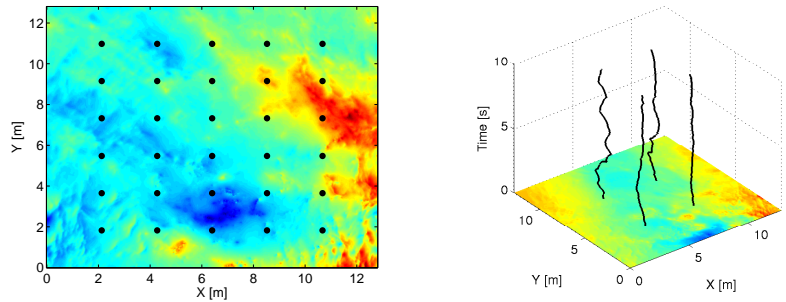

FIGURE 11. Experiment III (Crimea). Left: Location of the virtual probes. Right: Illustration of extracted time series at probe points within the space-time volume $Z(x, y, t)$.

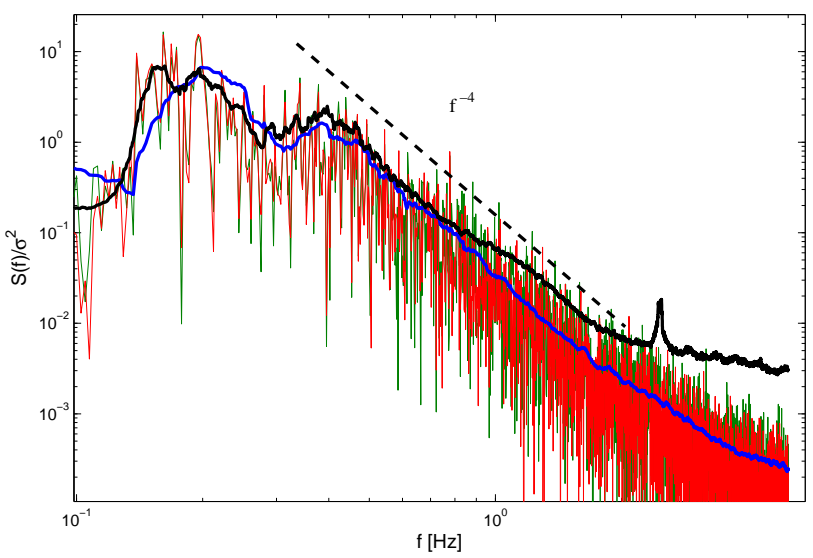

FIGURE 12. Experiment III (Crimea). Normalized frequency spectrum $\left(\sigma^{2}\right.$ is the variance of the wave surface) averaged over all virtual probes (blue line) and estimated counterpart using classical epipolar method (black line). Note that the Nyquist frequency (half of the sampling frequency) is $5 \mathrm{~Hz}$, according to the snapshot (e.g. frame) rate.

pend upon the first minimum of the wave covariance $\psi(T)$. In particular the mean values of $c$ and $\psi^{*}$ over the time series ensemble are $c \approx 1$ and $\psi^{*} \approx 0.52$.

\section{CONCLUSIONS}

Variational stereo is more powerful, flexible, and rigorous, albeit computationally expensive, than earlier traditional, imagebased stereo methods founded on epipolar line search. Therefore, we follow this research path by developing a variational stereo method for the case of smooth surfaces representable in the form of a graph supporting a smooth radiance function. We successfully apply this method to reconstruct the surface of the ocean. In future research we plan to elaborate on better choices for the regularizers as well as new ones that include global and/or local properties of the dynamics of ocean waves such as statistical distribution of wave heights, the wave equation, etc.

Besides the simple snapshot-by-snapshot sequential tempo-

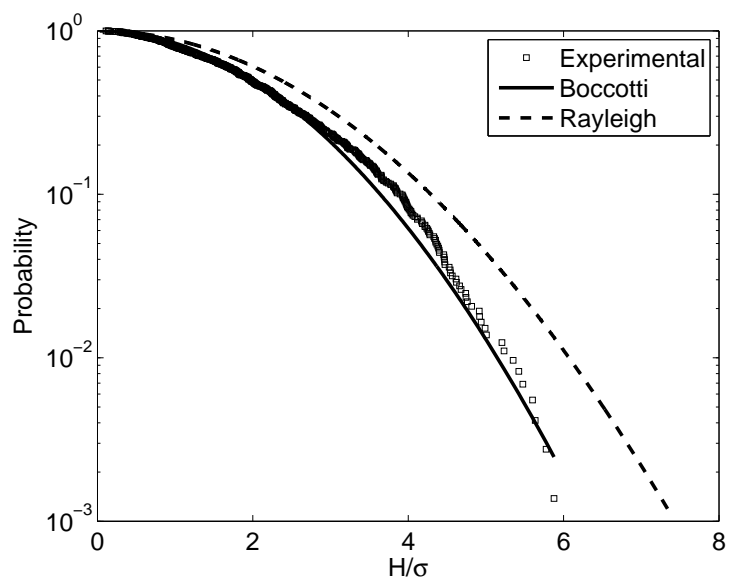

FIGURE 13. Experiment III (Crimea). Wave height exceedance probability estimated from all time series at virtual probes, compared to Rayleigh's distribution and Boccotti's distribution (19) ( $\sigma$ is the standard deviation of the wave surface).

ral processing used in the experiments, the variational framework allows for better ways to enforce space-time coherence of the reconstructed surface. This topic is now under investigation. Preliminary research shows that VWASS is a promising remotesensing observational technology with a broader impact on ocean engineering since it will enrich the understanding of the oceanic sea states and wave statistics, enabling improved designs of offshore structures and platforms.

\section{ACKNOWLEDGMENT}

Research supported by ONR grant BAA 09-012: "Ocean Wave Dissipation and energy Balance (WAVE-DB): toward reliable spectra and first breaking statistics".

\section{REFERENCES}

[1] Ma, Y., Soatto, S., Kosecka, J., and Sastry, S., 2003. An Invitation to 3D Vision: From Images to Geometric Models. Springer Verlag.

[2] Hartley, R. I., and Zisserman, A., 2004. Multiple View Geometry in Computer Vision, second ed. Cambridge University Press.

[3] Lu, Y., Zhang, J., Wu, Q., and Li, Z.-N., 2004. "A survey of motion-parallax-based 3-d reconstruction algorithms". Systems, Man, and Cybernetics, Part C: Applications and Reviews, IEEE Transactions on, 34(4), nov., pp. $532-548$.

[4] Seitz, S. M., Curless, B., Diebel, J., Scharstein, D., and Szeliski, R., 2006. "A comparison and evaluation of multiview stereo reconstruction algorithms". In CVPR 2006, Vol. 1, pp. 519-528.

[5] Benetazzo, A., Dec. 2006. "Measurements of short wa- 
ter waves using stereo matched image sequences". Coastal Engineering, 53(12), pp. 1013-1032.

[6] Wanek, J. M., and Wu, C. H., Apr. 2006. "Automated trinocular stereo imaging system for three-dimensional surface wave measurements". Ocean Engineering, 33(5-6), pp. 723-747.

[7] Faugeras, O. D., and Keriven, R., 1998. "Variational principles, surface evolution, pdes, level set methods, and the stereo problem". IEEE Trans. on Image Processing, 7(3), pp. 336-344.

[8] Yezzi, A., and Soatto, S., 2003. "Stereoscopic segmentation". International Journal of Computer Vision, 53(1), June, pp. 31-43.

[9] Jin, H., 2003. "Variational methods for shape reconstruction in computer vision". $\mathrm{PhD}$ thesis, Washington University, St. Louis, MO, USA. Director: Soatto, Stefano.

[10] Alvarez, L., Deriche, R., Sánchez, J., and Weickert, J., 2002. "Dense disparity map estimation respecting image discontinuities : A pde and scale-space based approach". $J$. of Visual Comm. and Image Rep., 13, pp. 3-21.

[11] Schumacher, A., 1939. "Stereophotogrammetrische wellenaufnahmen. wiss ergeb. dtsch. atlant. exped. forschungs vermessung. meteor 1925-1927". Ozeanographische Sonderuntersuchungen, Erste Lieferung,.

[12] Coté, L., Davis, J., Marks, W., McGough, R., Mehr, E., Pierson, W., Ropek, J., Stephenson, G., and Vetter, R., 1960. "The directional spectrum of wind generated sea as determined from data obtained by the stereo wave observation project". Metereological paper, 2(6).

[13] Sugimori, Y., 1975. "A study of the application of the holographic method to the determination of the directional spectrum of ocean waves". Deep Sea Research and Oceanographic Abstracts, 22(5), pp. 339 - 342, IN17-IN23, 343350.

[14] Barber, N., 1954. "Finding the direction of travel of sea waves". Nature, 154, p. 10481049.

[15] Holthuijsen, L. H., 1983. "Observations of the directional distribution of ocean-wave energy in fetch-limited conditions". J. of Physical Oceanography, 13(2), pp. 191-207.

[16] Shemdin, O., Tran, H., and Wu, S., 1988. "Directional measurements of short ocean waves with stereography". $J$. Geophys. Res., 93, pp. 13891-13901.

[17] Shemdin, O., and Tran, H., 1992. "Measuring short surface waves with stereography". Photogrammetric Engineering and Remote Sensing, 58(311-316).

[18] Holland, K., Holman, R., Lippmann, T., Stanley, J., and Plant, N., 1997. "Practical use of video imagery in nearshore oceanographic field studies". IEEE J. of Oceanic Engineering, 22(1), jan., pp. 81 -92.

[19] Santel, F., Linder, W., and Heipke, C., 2004. "Stereoscopic 3d-image sequence analysis of sea surfaces". In ISPRS
Commission V Symposium, Vol. 35, pp. 708-712.

[20] Gallego, G., Benetazzo, A., Yezzi, A., and Fedele, F., 2008. "Wave statistics and spectra via a variational wave acquisition stereo system". In OMAE. Lisbon, Portugal.

[21] MacHutchon, K., and Liu, P., 2007. "Measurement and analysis of ocean wave fields in four dimensions". In OMAE, Vol. 1, pp. 923-927.

[22] Stagonas, D., and Muller, G., 2007. "Wave field mapping with particle image velocimetry". Ocean Engineering, 34(11-12), pp. 1781-1785.

[23] Hao, K.-R., and Ding, Y.-S., 2008. "Trinocular matching realized by a monocular stereovision sensor for parallel manipulator". In ICARCV, pp. 1436-1441.

[24] Hsiao, Y.-H., and Huang, M.-C., 2009. "Application of active contour model in tracking sequential nearshore waves". China Ocean Engineering, 23(12), pp. 251-266.

[25] Fedele, F., Gallego, G., Yezzi, A., and Benetazzo, A., 2008. "Understanding extreme waves via a variational wave acquisition stereo system". In Workshop ROGUE WAVES. Oct 13-15, Brest, France.

[26] Briggs, W. L., Henson, V. E., and McCormick, S. F., 2000. A Multigrid Tutorial, Second Edition. SIAM.

[27] Zakharov, V. E., 1999. "Statistical theory of gravity and capillary waves on the surface of a finite-depth fluid". Eur. J. Mech. B - Fluids, 18(3), pp. 327-344.

[28] Phillips, O., 1958. "The equilibrium range in the spectrum of wind-generated waves". J. of Fluid Mechanics, 4(4), pp. 426-434.

[29] Dugan, J. P., Piotrowski, C. C., and Williams, J. Z., 2001. "Water depth and surface current retrievals from airborne optical measurements of surface gravity wave dispersion”. Journal of Geophysical Research, 106(C8), August, pp. 16903-16915.

[30] Hauser, D., Kahma, K. K., Krogstad, H. E., Lehner, S., Monbaliu, J., and Wyatt, L. R., 2003. European COST Action 714 (Measuring and Analysing the Directional Spectrum of Ocean Waves). European Science Fundation.

[31] Boccotti, P., 2000. Wave Mechanics for Ocean Engineering. Elsevier Oceanography Series. Elsevier, Oxford.

[32] Fedele, F., and Tayfun, A., 2009. "On nonlinear wave groups and crest statistics". J. of Fluid Mechanics, 620, pp. 221-239. 